Pacific Journal of Mathematics

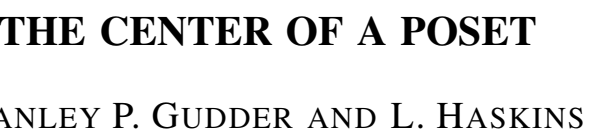




\title{
THE CENTER OF A POSET
}

\author{
S. GUdDER AND L. HASKINS
}

\section{The definition of neutral element is extended in a natural way from lattices to posets. The centers of posets of varying degrees of generality are then characterized.}

The center of a poset. In [1], G. Birkhoff states that the factorization of a poset with universal bounds is best analyzed by considering its center. He characterizes the center of a lattice as its set of complemented, neutral elements and asks (Problem 7, page 78) if the concept of neutral element can be extended to posets. We will generalize the definition of neutral element in such a way as to be able to extend his theorem on the center of a lattice to posets. For example we shall show that an element is in the center of a poset if and only if it is complemented and satisfies a generalized associative and distributive law and that an element is in the center of a multilattice if and only if it is complemented and satisfies a generalized distributive law. Other approaches to the factorization of a poset are possible (cf. [2]). However, our approach gives a direct generalization of Birkhoff's result.

Let $P$ be a poset with universal bounds 0 and 1 . If $A \cong P$ define

$$
\begin{aligned}
& U(A)=\{x \in P: x \geqq y \text { for some } y \in A\}, \\
& L(A)=\{x \in P: x \leqq y \text { for some } y \in A\}, \\
& M(A)=\{x \in A: y>x \text { for no } y \in A\}, \\
& m(A)=\{x \in A: y<x \text { for no } y \in A\} .
\end{aligned}
$$

Notice it follows from these definitions that $U(\phi)=L(\phi)=M(\phi)=$ $m(\phi)=\phi . \quad$ Let $S(P)$ be the power set on $P$. Then $u, l: S(P) \times S(P) \rightarrow$ $S(P)$ are defined by $A l B=M[L(A) \cap L(B)]$ and $A u B=m[U(A) \cap$ $U(B)]$. For convenience we write $x$ for the singleton set $\{x\}$. Thus if $P$ is a lattice $x l y=x \wedge y$ and $x u y=x \vee y$. An element $e \in P$ is central or (in the center) if $P=X \cdot Y$ where $X, Y$ are posets with 0 and 1 and $e=(0,1)$ or $(1,0)$. We denote the center of $P$ by $Z(P)$. An element $e \in P$ is complemented if there is an $e^{\prime} \in P$ such that $e u e^{\prime}=1$ and $e l e^{\prime}=0$.

To extend the concept of neutrality to posets, in a natural way, we must define the distributive laws for posets. Consider the following equations:

$$
\begin{aligned}
& e u(x l y)=(e u x) l(e u y), \\
& x u(e l y)=(x u e) l(x u y),
\end{aligned}
$$


and dually

$$
\begin{aligned}
& e l(x u y)=(e l x) u(e l y), \\
& x l(e u y)=(x l e) u(x l y) .
\end{aligned}
$$

We say that an element $e \in P$ is weakly distributive if (D1), (D4) hold whenever, $x l y \neq \phi$ and (D2), (D3) hold whenever $x u y \neq \phi$. We say that $e$ is distributive if $e$ is weakly distributive and (D2) holds whenever $x l e=y l e$ and $x u e=y u e$. An element $e$ is strongly distributive if (D1)-(D4) hold for all $x, y \in P$. In lattices these three types of distributive elements coalesce to the concept of neutral element.

An element $e \in P$ is associative if $x \leqq e, y l e=0$ imply $s u(x u y)=$ $(s u x) u y$ for all $s \in P$ and dually $x \geqq e, y u e=1$ imply $s l(x l y)=$ $(s l x) l y$ for all $s \in P$. Of course, if $P$ is a lattice all elements are associative. This need not be the case for posets.

Notice if $e$ is complemented and distributive then the complement is unique. Indeed, if $e^{\prime}, e^{\prime \prime}$ are complements of $e$ then since $e^{\prime} l e=$ $e^{\prime \prime} l e$ and $e^{\prime} u e=e^{\prime \prime} u e$ we can apply (D2) to obtain

$$
e^{\prime}=e^{\prime} u\left(e l e^{\prime \prime}\right)=\left(e^{\prime} u e\right) l\left(e^{\prime} u e^{\prime \prime}\right)=e^{\prime} u e^{\prime \prime} .
$$

Thus $e^{\prime} \geqq e^{\prime \prime}$ and by symmetry $e^{\prime} \leqq e^{\prime \prime}$.

THEOREM. An element $e$ is in $Z(P)$ if and only if $e$ is associative, complemented and distributive.

Proof. For necessity, suppose $e \in Z(P)$ and $e=(1,0)$ with respect to some factorization $P=X \cdot Y$. For $x, y \in P$ let $x=\left(x_{1}, x_{2}\right), y=\left(y_{1}, y_{2}\right)$ where $x_{1}, y_{1} \in X, x_{2}, y_{2} \in Y$. If $z \in x l y$, then $z_{1} \leqq x_{1}, y_{1}$ and $z_{2} \leqq x_{2}, y_{2}$. If there exists a $w_{1}$ such that $z_{1}<w_{1} \leqq x_{1}, y_{1}$ then $\left(z_{1}, z_{2}\right)<\left(w_{1}, z_{2}\right) \leqq$ $\left(x_{1}, x_{2}\right),\left(y_{1}, y_{2}\right)$ a contradiction. Thus $z_{1} \in x_{1} l y_{1}$ and $z_{2} \in x_{2} l y_{2}$. The argument reverses so $z \in x l y$ if and only if $z_{1} \in x_{1} l y_{1}$ and $z_{2} \in x_{2} l y_{2}$. We now show that $e$ is weakly distributive. If $x l y \neq \phi$ then

$$
\begin{aligned}
e u(x l y) & =m\left[U(1,0) \cap U\left\{\left(z_{1}, z_{2}\right): z_{i} \in x_{i} l y_{i}\right\}\right] \\
& =m\left\{(1, v): v \geqq z_{2} \in x_{2} l y_{2}\right\} \\
& =\left\{\left(1, z_{2}\right): z_{2} \in x_{2} l y_{2}\right\}=M\left\{(1, v): v \leqq x_{2}, y_{2}\right\} \\
& =M\left[L\left(1, x_{2}\right) \cap L\left(1, y_{2}\right)\right]=M[L(e u x) \cap L(e u y)] \\
& =(e u x) l(e u y) .
\end{aligned}
$$

If $x u y \neq \phi$ then

$$
\begin{aligned}
x u(e l y) & =m\left[U(x) \cap U\left\{\left(z_{1}, z_{2}\right): z_{i} \in e_{i} l y_{i}\right\}\right] \\
& =m\left[U\left(x_{1}, x_{2}\right) \cap U\left(y_{1}, 0\right)\right]=\left\{\left(v_{1}, x_{2}\right): v_{1} \in x_{1} u y_{1}\right\} \\
& =M\left\{\left(w_{1}, w_{2}\right): w_{1} \leqq v_{1} \in x_{1} u y_{1}, w_{2} \leqq x_{2}\right\} \\
& =M\left[L\left(1, x_{2}\right) \cap L\left\{\left(v_{1}, v_{2}\right): v_{i} \in x_{i} u y_{i}\right\}\right] \\
& =M[L(x u e) \cap L(x u y)]=(x u e) l(x u y) .
\end{aligned}
$$


The dual statements follow in a similar manner so $e$ is weakly distributive. To show $e$ is distributive suppose $x l e=y l e$ and $x u e=y u e$. It follows that $x=y$ so clearly $x u y \neq \phi$ and (D2) holds. If $x \leqq e$ and $y l e=0$ then $x=\left(x_{1}, 0\right)$ and $y=\left(0, y_{2}\right)$ so

$$
\begin{aligned}
(s u x) u y & =\left\{\left(r, s_{2}\right): r \in s_{1} u x_{1}\right\} u\left(0, y_{2}\right) \\
& =\left\{(r, t): r \in s_{1} u x_{1}, t \in s_{2} u y_{2}\right\} \\
& =\left(s_{1}, s_{2}\right) u\left(x_{1}, y_{2}\right)=s u(x u y) .
\end{aligned}
$$

The dual is similar so $e$ is associative. It is clear that $(0,1)$ is a complement for $e$.

We divide the proof of sufficiency into seven steps. Suppose $e$ is associative, complemented and distributive.

(1) We first show that $e \vee x$ and $e \wedge x$ exist for all $x \in P$. Now $e u x \neq \phi$ since otherwise $e=e u(x l 0)=(e u x) l(e u 0)=\phi l e=\phi$, contradiction. If $e \vee x$ does not exist there are distinct elements $z, w \in P$ with $z, w \in e u x$ since otherwise $e u x$ is a singleton set and if $s \geqq e, x$ we have $(e u x) l s=(e l s) u(x l s)=e u x$ so $e u x \leqq s$ which would imply $e u x=e \vee x$. Now

$$
z=z l(e u x)=(z l e) u(z l x)=e u x \supseteqq\{z, w\}
$$

which is impossible. Thus $e \vee x$ exists and the existence of $e \wedge x$ follows dually.

(2) We now show that el $A=M\{e \wedge a: a \in A\}$. By definition el $A=M[L(e) \cap L(A)]$. If $x \in e l A$ then $x \leqq e$ and $x \leqq a$ for some $a \in A$. If there exists $y \leqq e, a$ and $x<y$ this would contradict the maximality of $x$ in $L(e) \cap L(A)$, so $x \in e l a$ and $x=e \wedge a$. If $z=e \wedge a_{1}$ for some $a_{1} \in A$ then $z \in L(e) \cap L(A)$ so $z \ngtr x$. Hence $x \in M\{e \wedge a: a \in A\}$. Conversely, if $x \in M\{e \wedge a: a \in A\}$ then $x \in L(e) \cap L(A)$. Suppose $y \in L(e) \cap$ $L(A)$ and $y>x$. Then $y \leqq e, a$ for some $a \in A$ so $y \leqq e \wedge a$ which implies $x<e \wedge a$, a contradiction. Hence $x \in M[L(e) \cap L(A)]=e l A$. That e $u A=m\{e \vee a: a \in A\}$ follows dually.

Let $\phi_{e}: P \rightarrow L(e) \cdot U(e)$ be defined by $\phi_{e}(x)=(x \wedge e, x \vee e)$.

(3) To show $\phi_{e}$ is injective, suppose $\phi_{e}(x)=\phi_{e}(y)$. Then $x \wedge e=$ $y \wedge e, x \vee e=y \vee e$. It follows from (D2) that

$$
\begin{aligned}
y & =(y \wedge e) u y=(x \wedge e) u y=(x u y) l(e \vee y) \\
& =(x u y) l(e \vee x)=(y \wedge e) u x=(x \wedge e) u x=x .
\end{aligned}
$$

(4) If $x \leqq y$ then $x \wedge e \leqq y \wedge e$ and $x \vee e \leqq y \vee e$ so $\phi_{e}$ is order preserving.

(5) We now show that $d \wedge e^{\prime}$ exists if $d \geqq e$. By associativity, ol $\left(d l e^{\prime}\right)=($ old $) l e^{\prime}=0$ so $d l e^{\prime} \neq \phi$. Suppose $s, t \in d l e^{\prime}$. By (2) $m\left\{e \vee w: w \in d l e^{\prime}\right\}=e u\left(d l e^{\prime}\right)=e u d=d$. Since $d \geqq e, x, t$ we have 
$d \geqq e \vee s, e \vee t$. Hence $d=e \vee s=e \vee t$. Since $s, t \leqq e^{\prime}$ we have $e \wedge s, e \wedge t \leqq e \wedge e^{\prime}=0$ so $e \wedge s=e \wedge t=0$. Hence $\phi_{e}(s)=\phi_{e}(t)$ so by (3) $s=t$. Thus $d l e^{\prime}$ is a singleton set. If $z \leqq d, e^{\prime}$ then by associativity $z l\left(d l e^{\prime}\right)=(z l d) l e^{\prime}=z$ so $z \leqq d l e^{\prime}$ and $d l e^{\prime}=d \wedge e^{\prime}$.

(6) We now show that $\phi_{e}$ is bijective. Let $(c, d) \in L(e) \cdot U(e)$. We shall prove that $x \equiv c u\left(d \wedge e^{\prime}\right)=c \vee\left(d \wedge e^{\prime}\right)$ and that $e \wedge x=c$, $e \vee x=d$. Since $c \leqq e$ and $\left(d \wedge e^{\prime}\right) l e=0$, applying associativity $1 u\left[c u\left(d l e^{\prime}\right)\right]=(1 u c) u\left(d l e^{\prime}\right)=1$ so $c u\left(d l e^{\prime}\right) \neq \phi$. If $s, t \in c u\left(d \wedge e^{\prime}\right)$ then $s, t \geqq c$ and since $e \geqq c$ we have $e \wedge s, e \wedge t \geqq c$. Applying (2) we conclude that $e \wedge s=e \wedge t=c$. Now $s, t \geqq d \wedge e^{\prime}$ and hence $s \vee e, t \vee e \geqq\left(d \wedge e^{\prime}\right) \vee e=(d \vee e) \wedge\left(e^{\prime} \vee e\right)=d \vee e=d$. Now

$$
\begin{aligned}
c u\left(d \wedge e^{\prime}\right) & =(c \wedge e) u\left(d \wedge e^{\prime}\right)=\left[c u\left(d \wedge e^{\prime}\right)\right] l\left[e u\left(d \wedge e^{\prime}\right)\right] \\
& =\left[c u\left(d \wedge e^{\prime}\right)\right] l d,
\end{aligned}
$$

and hence $s, t \leqq d$. Since $e \leqq d$ it follows that $s \vee e, t \vee e \leqq d$ and hence $s \vee e=t \vee e=d$. Applying (3), $s=t=x$ and $x \wedge e=c$, $x \vee e=d$ so $\phi_{e}$ is bijective. If $z \geqq c, d \wedge e^{\prime}$, then by associativity $z u\left[c u\left(d \wedge e^{\prime}\right)\right]=(z u c) u\left(d \wedge e^{\prime}\right)=z$ so $z \geqq c u\left(d \wedge e^{\prime}\right)$ and $c u\left(d \wedge e^{\prime}\right)=$ $c \vee\left(d \wedge e^{\prime}\right)$.

(7) To show $\phi_{e}^{-1}$ is order preserving suppose $(a, b),(c, d) \in L(e) \cdot U(e)$ and $(a, b) \leqq(c, d)$. Then by (6) $\phi_{e}^{-1}(a, b)=a \vee\left(b \wedge e^{\prime}\right)$ and $\phi_{e}^{-1}(c, d)=$ $c \vee\left(d \wedge e^{\prime}\right)$. But clearly $a \vee\left(b \wedge e^{\prime}\right) \leqq c \vee\left(d \wedge e^{\prime}\right)$

It follows that $P$ is isomorphic to $L(e) \cdot U(e)$ and the proof is complete.

CoRollary. If e is associative, complemented and strongly distributive, then $e \in Z(P)$.

The converse of the corollary does not hold. Specifically, if $e \in Z(P)$ then although $e$ must be associative, complemented and distributive, $e$ need not be strongly distributive. For example, let $X=\{0,1\}$ and let $Y$ be a poset with 0,1 and two elements $x, y$ satisfying $x l y=\phi$. If $P=Y \cdot X$ then $e=(1,0) \in Z(P)$. However,

$$
e u[(x, 0) l(y, 0)]=e u \phi=\phi \neq e=e l e=[e u(x, 0)] l[e u(y, 0)]
$$

so $e$ is not strongly distributive. This difficulty can not be eliminated by making the convention $U(\phi)=L(\phi)=P$. Indeed, in this case if we let $P=X \cdot Y$ then $e=(1,0) \in Z(P)$, but

$$
e u[(0, x) l(0, y)]=e u \phi=e \neq \phi=(1, x) l(1, y)=[e u(0, x)] l[e u(0, y)]
$$

so again $e$ is not strongly distributive.

For the posets in the next corollary the three types of distributivity are the same. 
Corollary. Let $P$ satisfy $x u y, x l y \neq \phi$ for all $x, y \in P$. Then $e \in Z(P)$ if and only if $e$ is associative, complemented and strongly distributive.

A multilattice is a poset for which $s \leqq x, y$ implies there exist $z \in x l y$ such that $s \leqq z$ and $t \geqq x, y$ implies there is a $w \in x u y$ such that $t \geqq w$. Multilattices are still vast generalizations of lattices; in particular, any poset with no infinite chains is a multilattice. In a multilattice the associative laws always hold so every element is associative. Indeed, if $z \in s u(x u y)$ then $z \geqq s, z \geqq z_{1} \in x u y$ and $z$ is minimal. Then $z \geqq s, x, y$ so $z \geqq z_{2} \in s u x$. Suppose there is $z_{3} \geqq y$, $z_{3} \geqq z_{4} \in s u x$, with $z>z_{3}$. Then $z_{3} \geqq s, z_{3} \geqq z_{5} \in x u y$ which contradicts the minimality of $z$. Thus $z \in(s u x) u y$. By symmetry $s u(x u y)=$ $(s u x) u y$ and the other associative law holds similarly. Our next result gives the most direct generalization of Birkhoff's theorem $[1$, page 69].

Corollary. If $P$ is a multilattice with 0,1 then $e \in Z(P)$ if and only if $e$ is complemented and strongly distributive.

Of course, in this case the three types of distributivity are the same.

\section{REFERENCES}

1. G. Birkhoff, Lattice Theory, Amer. Math. Soc. Colloq. Publ. XXV, Providence, R. I., 1967.

2. V. Novak and J. Schmidt, Direct decompositions of partially ordered sets with zero,

J. Reine Angew. Math., 239/240 (1969), 97-108.

Received January 3, 1973.

UNIVERSITY OF DENVER

AND

CARLTON UNIVERSity 



\section{PACIFIC JOURNAL OF MATHEMATICS}

EDITORS

RICHARD ARENS (Managing Editor)

University of California

Los Angeles, California 90024

R. A. Beaumont

University of Washington

Seattle, Washington 98105

J. DugundJI

Department of Mathematics

University of Southern California

Los Angeles, California 90007

D. Gilbarg and J. Milgram

Stanford University

Stanford, California 94305

\section{ASSOCIATE EDITORS}

E. F. BECKENBACH

B. H. NeumanN

F. WOLF

K. YOSHIDA

\section{SUPPORTING INSTITUTIONS}

UNIVERSITY OF BRITISH COLUMBIA CALIFORNIA INSTITUTE OF TECHNOLOGY

UNIVERSITY OF CALIFORNIA

MONTANA STATE UNIVERSITY

UNIVERSITY OF NEVADA

NEW MEXICO STATE UNIVERSITY

OREGON STATE UNIVERSITY

UNIVERSITY OF OREGON

OSAKA UNIVERSITY

\author{
UNIVERSITY OF SOUTHERN CALIFORNIA \\ STANFORD UNIVERSITY \\ UNIVERSITY OF TOKYO \\ UNIVERSITY OF UTAH \\ WASHINGTON STATE UNIVERSITY \\ UNIVERSITY OF WASHINGTON \\ $\stackrel{*}{*} \stackrel{*}{*}{ }^{*}{ }^{*}{ }^{2}$ AMERICAN MATHEMATICAL SOCIETY \\ NAVAL WEAPONS CENTER
}




\section{Pacific Journal of Mathematics}

\section{Vol. 52, No. $1 \quad$ January, 1974}

David R. Adams, On the exceptional sets for spaces of potentials ............ 1

Philip Bacon, Axioms for the Čech cohomology of paracompacta ............ 7

Selwyn Ross Caradus, Perturbation theory for generalized Fredholm operators ..... 11

Kuang-Ho Chen, Phragmén-Lindelöf type theorems for a system of nonhomogeneous equations ............................ 17

Frederick Knowles Dashiell, Jr., Isomorphism problems for the Baire classes .......

M. G. Deshpande and V. K. Deshpande, Rings whose proper homomorphic images are right subdirectly irreducible . . . . . . . . . . . . . . . . . . . . . . . . .

Mary Rodriguez Embry, Self adjoint strictly cyclic operator algebras .............

Paul Erdős, On the distribution of numbers of the form $\sigma(n) / n$ and on some related

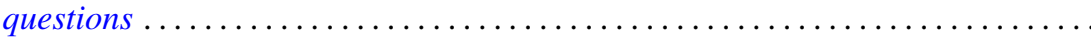

Richard Joseph Fleming and James E. Jamison, Hermitian and adjoint abelian

operators on certain Banach spaces ............................

Stanley P. Gudder and L. Haskins, The center of a poset .................. 85

Richard Howard Herman, Automorphism groups of operator algebras . . . ........

Worthen N. Hunsacker and Somashekhar Amrith Naimpally, Local compactness of families of continuous point-compact relations ....................

Donald Gordon James, On the normal subgroups of integral orthogonal groups ....

Eugene Carlyle Johnsen and Thomas Frederick Storer, Combinatorial structures in

loops. II. Commutative inverse property cyclic neofields of prime-power

order.

Ka-Sing Lau, Extreme operators on Choquet simplexes . . . . . . . . . . . . . . 129

Philip A. Leonard and Kenneth S. Williams, The septic character of 2, 3, 5 and $7 \ldots 143$

Dennis McGavran and Jingyal Pak, On the Nielsen number of a fiber map ........ 149

Stuart Edward Mills, Normed Köthe spaces as intermediate spaces of $L_{1}$ and

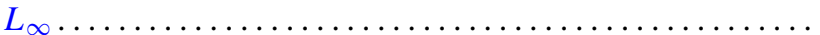

Philip Olin, Free products and elementary equivalence. .

Louis Jackson Ratliff, Jr., Locally quasi-unmixed Noetherian rings and ideals of the principal class.

Seiya Sasao, Homotopy types of spherical fibre spaces over spheres ...

Helga Schirmer, Fixed point sets of polyhedra ...

Kevin James Sharpe, Compatible topologies and continuous irreducible

representations.

Frank Siwiec, On defining a space by a weak base . . . . . . . . . . . . . . . 233

James McLean Sloss, Global reflection for a class of simple closed curves ....... 247

M. V. Subba Rao, On two congruences for primality . .

Raymond D. Terry, Oscillatory properties of a delay differential equation of even

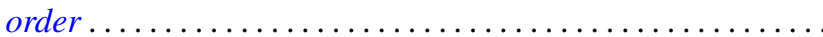

Joseph Dinneen Ward, Chebyshev centers in spaces of continuous functions . .

Robert Breckenridge Warfield, Jr., The uniqueness of elongations of Abelian

groups...

V. M. Warfield, Existence and adjoint theorems for linear stochastic differential

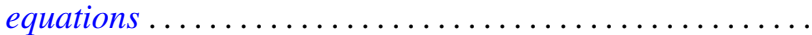

